\title{
Lifestyle Counseling for Patients with Type 2 Diabetes in the Southwest of Saudi Arabia: An Example of Healthcare Delivery Inequality Between Different Healthcare Settings
}

\author{
Ibrahim M Gosadi (D) \\ Department of Family and Community \\ Medicine, Faculty of Medicine, Jazan \\ University, Jazan, 8262I, Saudi Arabia
}

Purpose: Adherence to a healthy lifestyle can have a positive impact on the course of diabetes management. This investigation aims to study lifestyle counseling provision among patients with type 2 diabetes in Saudi Arabia and the delivery of lifestyle counseling within different healthcare settings.

Methods: This cross-sectional investigation was conducted in the Jazan region in Saudi Arabia. Data were collected using a semi-structured questionnaire during phone interviews. The questionnaire measured items related to the demographics of the patients, diabetes diagnoses, and follow-up locations. The items pertaining to lifestyle counseling were related to whether the patients were given an assessment of dietary and physical activity levels, receipt of a personalized lifestyle change plan, and smoking cessation therapy among smokers. Odds ratios (ORs) were calculated to estimate the probability of receiving the lifestyle counseling items in primary healthcare centers (PHCs) compared to other healthcare facilities.

Results: A total of 461 patients diagnosed with diabetes were identified. The development of symptoms or complications related to diabetes was the main cause of diagnosis (63\%). Sixtysix patients reported not attending a follow-up at any healthcare facility. The ORs of receiving a lifestyle plan and having daily caloric needs calculated were higher among patients who followed up at hospitals, diabetes centers or within the private sector compared to those who went to PHCs (ORs: 1.8 [1.1-2.8] and 2.7 [1.2-5.9] respectively). In all the identified healthcare settings, health education was mostly provided by the treating physicians, indicating the limited role of nutritionists or health educationists.

Conclusion: This study suggests a limited receipt of lifestyle counseling services for patients with type 2 diabetes in PHCs compared to other healthcare facilities. The majority of counseling services were provided by physicians, which indicates the limited interdisciplinary involvement of other health professionals, such as nutritionists and health educationists.

Keywords: diabetes, primary healthcare, health education, eating behavior, physical activity, smoking cessation, lifestyle counseling

\section{Introduction}

Lifestyle counseling can be defined as professional guidance and support to aid individuals modify their lifestyle. Healthy lifestyle counseling aiming to reduce metabolic risk factors, including diabetes, focuses on modifying behaviors related to unhealthy diet, tobacco use, physical inactivity, and the harmful use of alcohol. ${ }^{1}$
Correspondence: Ibrahim M Gosadi Faculty of Medicine, Jazan University, P.O. Box: 2349, Jazan, 8262I, Saudi Arabia

Tel+966562I377II

Email gossady@hotmail.com 
Diabetes is one of the most prevalent illnesses in the world $^{2}$ and is considered among the top ten worldwide causes of mortality. ${ }^{3}$ Due to the chronic nature of the disease and the high risk of developing several complications, diabetes causes a substantial direct and indirect cost for healthcare. Furthermore, the economic cost is augmented by the impact on productivity due to diabetes and its complications. $^{4,5}$

Although diabetes can have a severe impact on health, its early detection and adherence to a healthy lifestyle can have a positive impact on the course of the disease. According to the American Diabetes Association, the ramifications of the disease can be reduced by several preventive measures. These measures can be related to nutritional behavior, physical activity, smoking cessation, and preventive medications. ${ }^{6}$ Moreover, lifestyle modification is a core method for the management of patients with diabetes in addition to pharmacological therapy. ${ }^{7}$

Saudi Arabia is one of the countries where the high prevalence of diabetes has a significant effect. According to the International Diabetes Federation, more than four million Saudi adults have diabetes, constituting approximately $18.3 \%$ of the adult population, with the majority affected with type 2 diabetes. $^{8}$ Several investigations into hereditary and lifestyle aspects have been conducted to assess the contributing factors for the increased prevalence of diabetes, ${ }^{9}$ which is expected to grow among the Saudi population. ${ }^{10}$

Several guidelines have been produced to promote evidence-based practice for the prevention and management of diabetes. These guidelines can vary according to the country, patient groups, healthcare settings, and comorbidities. . $^{7-13}$ The Saudi Ministry of Health is one of the major contributors to healthcare services in Saudi Arabia, and it produced "The Saudi National Reference of Clinical Guidelines for Care of Diabetic Patients 2014" in an effort to enhance the quality of healthcare provided to patients with diabetes in the country. ${ }^{14}$ In these guidelines, several clinical recommendations are provided for the management of patients with diabetes, such as pharmacological therapies, health education, psychological and emotional well-being, weight loss, physical activity, advice on dietary behavior, and smoking cessation.

Pharmacological therapies prescribed for the management of diabetes can have a limited impact if they are not accompanied by strategies for the early identification of cases and effective lifestyle modification. Promoting a healthy lifestyle to patients with diabetes can be influenced by factors related to the quality of the provided healthcare and the patients' characteristics. A healthy lifestyle can be enhanced through health education on lifestyle change, weight management, promotion of physical activity and healthy dietary intake, and smoking cessation.

There are multiple local and international studies that have assessed the provision of lifestyle counseling for patients with chronic diseases, including diabetes. In a retrospective US study, which includes data from more than 184 million physicians visits for patients diagnosed with type 2 diabetes, hyperlipidemia, hypertension, or obesity, an assessment of the percentage of physicians who reported counseling on diet, exercise, weight reduction, or smoking cessation revealed that patients did not receive any type of diet or exercise counseling during half of the visits. ${ }^{15}$ Additionally, in a more recent U.S.-based study, it was concluded that the provision of lifestyle education for patients diagnosed with hyperlipidemia declined from $39.7 \%$ in 2005 to $22.4 \%$ in 2014 , while rates of using pharmacotherapy remained similar during the same period. ${ }^{16}$

Other studies assessed the provision of certain components of lifestyle counseling within PHC settings. A Dutch study explored the lifestyle counseling provision in routine primary care by means of videotaping lifestyle counseling sessions provided by physicians and practice nurses, which indicated a need for improvement in lifestyle counseling among these practitioners working in Dutch primary care settings. ${ }^{17}$ Additionally, a systematic review assessing physical activity counseling training in primary care identified four eligible studies, concluded a lack of evidence due to the limited number of studies, and emphasized the importance of establishing training programs in physical activity counseling in primary care residency programs. ${ }^{18}$ Furthermore, a Canadian study evaluating physical activity assessment and counseling provision among patients attending primary healthcare settings reported that nearly half of the patients had their physical activity level assessed and only $21.6 \%$ of them received physical activity counseling. ${ }^{19}$ Finally, a systematic review assessing smoking cessation counseling by primary care physicians identified 35 eligible studies and reported the presence of variability in the strategies used during the counseling. Considering the 5A's strategy for smoking cessation therapy (ask, advise, assess, assist, arrange), it was noted that physicians were more likely to engage in first strategy components (ask and advice) than the remaining components (assess, assist, arrange). ${ }^{20}$ 
Conflicting findings are identified regarding the provision of lifestyle counseling in PHC settings in Saudi Arabia. A study by Alhamdan et al, conducted in 15 randomly selected PHCs in the country's capital city of Riyadh, indicated that more than $80 \%$ of the selected centers reported a provision for counseling pertaining to physical activity, healthy eating, and tobacco use cessation. Nonetheless, their study indicated variability in the provision of lifestyle counseling services, suggesting a lack of a consistent guidelines for the provision of counseling services in PHC settings. ${ }^{21}$ In addition, in a review by Alahmad and Lobelo, which was published in 2018 and studies physical activity counseling in PHC settings in Saudi Arabia, it was concluded that despite the importance of the influence of physical activity on reducing the risk of chronic noncommunicable diseases, the data on physical activity counseling in PHC settings in Saudi Arabia are limited. ${ }^{22}$ Finally, a survey conducted by Jradi to assess smoking cessation counseling among 124 physicians form Riyadh reported that $68.6 \%$ of the physicians did not receive any form of smoking cessation counseling training and concluded there was limited involvement by the physicians in smoking cessation therapy. ${ }^{23}$

Patients with type 2 diabetes in Saudi Arabia can receive free healthcare services in primary, secondary and tertiary healthcare settings in addition to the private sector. Nonetheless, PHCs are considered the first providers of care for patients diagnosed with type 2 diabetes and provide healthcare to the majority of patients. Nonetheless, evidence concerning the provision of lifestyle counseling to patients with type 2 diabetes in the Saudi Arabia is currently limited and lacking in southwestern Jazan. This investigation aims to study the provision of lifestyle counseling among patients diagnosed with diabetes in the Jazan region and assess variations in its delivery according to the type of healthcare facility.

\section{Methods}

\section{Study Context}

This is a cross-sectional investigation conducted in the Jazan region in southwest Saudi Arabia between January and August 2020, which targets patients diagnosed with diabetes to assess their receipt of lifestyle counseling. This study is part of a project conducted to assess the utilization of preventive services for the control of chronic noncommunicable diseases in this region. These services include screening for obesity, diabetes, hypertension, and hypercholesterolemia, in addition to lifestyle counseling, health education and dietician services. Ethical approval to conduct the project was obtained from the Standing Committee for Scientific Research Ethics of Jazan University (approval number REC 40/3090) and administrative approval was obtained from the Directory of Public Health in Jazan. The study was conducted in accordance with the Declaration of Helsinki.

\section{Data Collection}

A data collection tool was developed after consulting the "Saudi National Reference of Clinical Guidelines for Care of Diabetic Patients 2014"14 and the "Saudi National Reference for Diabetes Mellitus Guidelines in Primary Health Care". 7 The developed semi-structured questionnaire measured items related to the patients' demographic characteristics, history of comorbidities with chronic noncommunicable diseases, duration of diabetes since diagnosis and how the diagnosis was performed, follow-up location, and lifestyle counseling received. The items pertaining to lifestyle counseling were related to whether the patients had their dietary and physical activity levels assessed by obtaining dieting history, identifying problematic eating behaviors, and obtaining weight and physical activity history. In addition, patients were asked whether a personalized lifestyle change plan was developed based on the initial assessment, including weight management with realistic weight-loss goals, personalized physical activity advice, and smoking cessation therapy among smokers.

The developed questionnaire was assessed by consultants in family medicine and epidemiology to evaluate the content validity by assessing the suitability of the content to measure the intended variables. Furthermore, the questionnaire was piloted on a sample of ten patients to test the face validity of the questionnaire by evaluating the clarity of the constructed items and the patients' ability to respond to the questions. The internal reliability of items measuring lifestyle counseling was assessed using Cronbach's alpha, which generated a reasonable internal consistency of 0.56 . Additionally, split-half reliability was administered and revealed a Spearman-Brown coefficient of 0.691 and a Guttman Split-half coefficient of 0.652.

After obtaining administrative approval from the Directory of Health in the Jazan region to conduct the study in clinical settings, the study was advertised to the attendees of conveniently selected PHCs in different 
governorates in the region. The data were collected during phone interviews, which were conducted by trained medical students. The training was conducted to ensure the consistency of the data collection process and to reduce the probability of measurement bias. To reduce the risk of selection bias, the data were collected through interviews to ensure the recruitment of patients who were unable to utilize self-administered questionnaires.

The patients that were interested in participating could contact the data collectors and arrange the interviews. Since participation was performed through phone calls, verbal informed consent was obtained before the start of the study, and patients were assured that their participation was voluntary and anonymous, and that they could withdraw at any time during the interview. At the end of the interview, the patients were asked to advertise the study to their neighbors, relatives, and friends to initiate snowball sampling, a non-random sampling method. This approach, though non-random, enabled the recruitment of patients diagnosed with diabetes who had stopped attending healthcare facilities after they were diagnosed with the disease. This study included patients who were diagnosed with type 2 diabetes in the Jazan region. Patients who had not been diagnosed with type 2 diabetes, did not complete the interview, and those who were under 18 years old were excluded from this investigation.

The study by Alhamdan et al reported that more than $80 \%$ of a selected sample of 15 PHCs in the Riyadh region provided lifestyle counseling services. However, their study was limited to the assessment of PHCs' infrastructure and provided services without the recruitment of any patients. $^{21}$ With the lack of national or regional data reporting the utilization of lifestyle counseling services, data from other international investigations were used to calculate the required sample size for the current investigation. In a study by Karr et al, based on a survey of office-based physicians visits in the United States, it was indicated that nutritional counseling was provided or ordered for $23.1 \%$ of visits by patients with diabetes, and physical activity counseling was provided or ordered for $15.2 \%{ }^{24}$ Based on these findings, we assumed that on average $20 \%$ of patients with diabetes in the Jazan region would have received some lifestyle counseling services during their visits. Using the SATCAL function of Epi Info, the sample size of the current survey was 424 patients with diabetes, assuming that $20 \%$ of the patients would have received lifestyle counseling services, with a $5 \%$ margin of error and a $99 \%$ confidence level.

\section{Data Analysis}

Statistical Package for the Social Sciences version 25 was used to perform the data analysis. The data were summarized utilizing frequencies and proportions for the binary and categorical data. For the continuous data, the mean and standard deviation (SD) were used to summarize the normally distributed data, and the median and interquartile range, were used to summarize the non-normally distributed data. The characteristics of lifestyle counseling reported by the participants were assessed according to follow-up location. The statistical variation of the frequencies and proportions of the reported receipt of lifestyle counseling was assessed according to the follow-up location, where the presence of a statistical difference was assessed via a chi-square test or Fisher Exact test. Similarly, the statistical variation of the frequencies and proportions of the reported receipt of lifestyle counseling was assessed according to the history of comorbidities, duration since diagnosis with diabetes and educational levels of the patients through chi-square test or Fisher Exact test. Finally, to measure the probability of receiving lifestyle counseling according to the healthcare establishment, the follow-up location was dichotomized into two binary variables where patients following up in PHCs were compared to patients following up at other healthcare facilities (including hospitals, diabetes centers, or within the private sector). The dichotomization of the follow-up location was based on the findings, which showed the majority of the patients following up at PHC clinics. This was investigated by performing univariate logistic regression to test for the odds of receiving lifestyle counseling services according to the follow-up location. A p-value of 0.05 was considered as a statistically significant value for the applied statistical test.

\section{Results}

The number of patients diagnosed with diabetes that were recruited in this investigation was 461. Sixty-four individuals were excluded for not meeting the inclusion criteria or completing the interview. Table 1 illustrates the demographic characteristics of the patients with diabetes, showing that more than half of the respondents were male ( 253 patients). The mean age of the patients was 54 years, and the majority of the patients were either illiterate $(27 \%)$ or holders of a university degree $(28 \%)$. Most the patients were living in owned 
Table I Demographics of 46I Patients Diagnosed with Diabetes from Jazan, Saudi Arabia

\begin{tabular}{|c|c|}
\hline Variables & \\
\hline Age in years: Mean [SD] & $54.2[13.7]$ \\
\hline \multicolumn{2}{|l|}{ Gender: Frequency [proportion] } \\
\hline Males & 253 [54.9\%] \\
\hline Females & 208 [45.1\%] \\
\hline \multicolumn{2}{|l|}{ Educational level: Frequency [proportion] } \\
\hline Illiterate & $125[27.1 \%]$ \\
\hline Elementary & $88[19.1 \%]$ \\
\hline Intermediate & $31[6.7 \%]$ \\
\hline Secondary & $86[18.7 \%]$ \\
\hline University & $|3|$ [28.5\%] \\
\hline \multicolumn{2}{|l|}{ Residence: Frequency [proportion] } \\
\hline Owned & 421 [91.3\%] \\
\hline Rented & $40[8.7 \%]$ \\
\hline \multicolumn{2}{|l|}{ Area: Frequency [proportion] } \\
\hline Urban & $194[42.1 \%]$ \\
\hline Rural & 267 [57.9\%] \\
\hline BMI*: Median [25-75 IQR] & $27.3[24.5-31.02]$ \\
\hline \multicolumn{2}{|c|}{ Distribution of comorbidities: Frequency [proportion] } \\
\hline Diabetes only & 217 [47.1\%] \\
\hline Diabetes, Dyslipidemia & $13[2.8 \%]$ \\
\hline Diabetes, Dyslipidemia, Obesity & $\mathrm{I}[0.2 \%]$ \\
\hline Diabetes, Hypertension & $165[35.8 \%]$ \\
\hline Diabetes, Hypertension, Dyslipidemia & $52[11.3 \%]$ \\
\hline Diabetes, Hypertension, Dyslipidemia, Obesity & $5[1.1 \%]$ \\
\hline Diabetes, Hypertension, Obesity & $4[0.9 \%]$ \\
\hline Diabetes, Obesity & $4[0.9 \%]$ \\
\hline \multicolumn{2}{|l|}{ Location of follow up: Frequency [proportion] } \\
\hline No follow up & $66[14.3 \%]$ \\
\hline PHCs & 259 [56.2\%] \\
\hline Hospitals & $63[13.7 \%]$ \\
\hline Diabetes center & $54[11.7 \%]$ \\
\hline Private sector & 19 [4.1\%] \\
\hline
\end{tabular}

Note: $* 3$ cases missing

residences (91\%) in rural areas (58\%). When the patients were asked about their latest body weight and height, their body mass index (BMI) was calculated and revealed that the median BMI level was 27 and about one-third of the patients had a BMI level of more than 30 , indicating a high prevalence of obesity in the sample.
Less than half of the patients diagnosed with diabetes had no diagnosed comorbidities. However, the remaining patients were diagnosed with another disease in addition to diabetes, with the most frequently reported comorbidity being hypertension. Nonetheless, it must be noted that when the patients were asked whether they were diagnosed as obese individuals by their physicians, only $3.1 \%$ of the patients reported being diagnosed as such, which conflicts with their estimated BMI levels that indicated a higher level of obesity. This can be partially explained by the probability that obesity was not referred to as a disorder, either by the physicians or by the patients themselves. When the patients were asked where they were attending the follow-up sessions, 66 patients (14\%) reported no follow-up attendance at any healthcare facility. Among those who reported attending a follow-up at healthcare facilities, the majority reported attending the follow-up at PHCs, followed by hospitals and diabetes centers.

Table 2 illustrates the method of identifying the disease among the respondents. The majority of the participants indicated that they were diagnosed with diabetes after the development of symptoms or complications related to diabetes (63\%). About a quarter of the sample (106 patients) learned that they had diabetes during laboratory investigations due to reasons other than diabetes. Only 22 patients $(5 \%)$ declared that they were diagnosed as patients with diabetes during screening campaigns. Finally, the mean disease duration from diagnoses for the recruited sample was 10 years (SD: 7.6 years).

Table 2 Reasons for Identifying the Development of Diabetes and the Duration of the Disease Since Diagnosis Among 46I Patients Affected with Diabetes in Jazan, Saudi Arabia

\begin{tabular}{|l|l|}
\hline Reasons: & $\begin{array}{l}\text { Frequency } \\
\text { [Proportion] }\end{array}$ \\
\hline $\begin{array}{l}\text { Identified during seeking healthcare after } \\
\text { appearance of symptoms or complications }\end{array}$ & 291 [63\%] \\
\hline $\begin{array}{l}\text { Identified accidently during investigations for } \\
\text { other reasons }\end{array}$ & $106[23 \%]$ \\
\hline Identified during screening campaigns & $22[4.8 \%]$ \\
\hline Identified during a routine follow up & $20[4.3 \%]$ \\
\hline Identified during pregnancy & $9[2 \%]$ \\
\hline None declared & $13[2.9 \%]$ \\
\hline Disease duration: Mean [SD]: I0 [7.6] & \\
\hline
\end{tabular}


Table 3 Characteristics of Lifestyle Counseling Delivery Reported by 461 Patients Diagnosed with Diabetes in Jazan, Saudi Arabia and Classified According to the Location of the Follow Up

\begin{tabular}{|c|c|c|c|c|c|}
\hline \multirow[t]{2}{*}{ Variables: Frequency [Proportion] } & \multicolumn{4}{|c|}{ Location of Follow Up } & \multirow[t]{3}{*}{$P$ value } \\
\hline & PHC & Hospital & Diabetes Center & Private Sector & \\
\hline Total sample & 259 & 63 & 54 & 19 & \\
\hline \multicolumn{6}{|c|}{ Person who delivered the health education to patients: } \\
\hline Physician & 113 [43.6\%] & $32[50.7 \%]$ & $32[59.2 \%]$ & II $[57.8 \%]$ & $0.001 * *$ \\
\hline Dietitian & I $[0.3 \%]$ & $2[3.1 \%]$ & $8[14.8 \%]$ & $3[15.7 \%]$ & \\
\hline Nurse/health educationist & $5[1.9 \%]$ & $2[3.1 \%]$ & $4[7.4 \%]$ & $0[0.0 \%]$ & \\
\hline \multicolumn{6}{|l|}{ Patients who received lifestyle change plan } \\
\hline & 57 [22.0\%] & 14 [22.2\%] & $26[48.1 \%]$ & $6[31.6 \%]$ & $0.001 * *$ \\
\hline \multicolumn{6}{|c|}{ Patients who had weight assessment and were advised to lose weight } \\
\hline & $102[39.4 \%]$ & 30 [47.6\%] & $31[57.4 \%]$ & $6[31.6 \%]$ & $0.05^{*}$ \\
\hline \multicolumn{6}{|c|}{ Patients who reported having their dietary habits assessed } \\
\hline & 77 [29.7\%] & 12 [19\%] & $29[53.7 \%]$ & $4[21.1 \%]$ & $0.001 * *$ \\
\hline \multicolumn{6}{|c|}{ Patients who were advised to reduce salt intake } \\
\hline & 122 [47.1\%] & 35 [55.6\%] & $25[46.3 \%]$ & $2[10.5 \%]$ & $0.005^{* *}$ \\
\hline \multicolumn{6}{|c|}{ Patients who reported having their daily caloric needs calculated } \\
\hline & $12[4.6 \%]$ & $6[9.5 \%]$ & $10[18.5 \%]$ & $0[0.0 \%]$ & $0.004 * *$ \\
\hline \multicolumn{6}{|c|}{ Patients who reported having their physical activity measured } \\
\hline & $6[2.3 \%]$ & $4[6.3 \%]$ & $2[3.7 \%]$ & $0[0.0 \%]$ & $0.302 * *$ \\
\hline \multicolumn{6}{|l|}{ Patients who were advised to exercise } \\
\hline & $197[76.1 \%]$ & $54[85.7 \%]$ & $44[81.5 \%]$ & 15 [78.9\%] & 0.379 \\
\hline \multicolumn{6}{|c|}{ Patients who reported being current smokers during interviews } \\
\hline & $21[8.1 \%]$ & 7 [1 $1.1 \%]$ & $4[7.4 \%]$ & I $[5.3 \%]$ & $0.804 * *$ \\
\hline \multicolumn{6}{|c|}{ Patients who reported being referred to smoking cessation clinic } \\
\hline & $8[3.1 \%]$ & $\mathrm{I}[1.6 \%]$ & $0[0.0 \%]$ & $0[0.0 \%]$ & 0.737 \\
\hline
\end{tabular}

Notes: *Chi-Square test. **Fisher Exact test.

Table 3 illustrates the characteristics of the lifestyle counseling delivery as reported by the patients according to the follow-up location. When the patients were asked about the healthcare professional who delivered health education about the disease, the majority of the respondents indicated that the education was delivered by treating physicians. The nutritionists' contributions were highest among diabetes centers and within the private sector. Only 13 patients indicated that they received health education from a nurse or health educationist, with none delivered within the private sector.
When the patients were asked about the delivery of advice concerning their lifestyle, the proportion of attendees who reported receiving a lifestyle change plan, advice related to weight loss, a dietary habits assessment, or a calculated evaluation of their daily caloric needs was higher among the diabetes center attendees compared to the attendees of the other healthcare facilities $(p<0.05)$. Although the majority of the patients were advised to engage in higher levels of physical activity, only 12 of them reported having their physical activity level measured by any means. This indicates a lack of goal- 
Table 4 Odds of Receiving Lifestyle Counseling Services Among Patients Following Up at PHCs to Patients Following Up at Other Healthcare Facilities

\begin{tabular}{|c|c|c|c|c|c|}
\hline Lifestyle Counseling Services & PHC [Reference] & Others & ORs & $95 \% \mathrm{Cls}$ & P value \\
\hline Total sample & 259 & 136 & & & \\
\hline \multicolumn{6}{|c|}{ Patients who received lifestyle change plan } \\
\hline & $57[22.0 \%]$ & $46[33.8 \%]$ & 1.8 & $1.1-2.8$ & 0.012 \\
\hline \multicolumn{6}{|c|}{ Patients who had weight assessment and were advised to lose weight } \\
\hline & 102 [39.4\%] & $67[49.3 \%]$ & 1.4 & $0.9-2.2$ & 0.060 \\
\hline \multicolumn{6}{|c|}{ Patients who reported having their dietary habits assessed } \\
\hline & 77 [29.7\%] & $45[33.1 \%]$ & I.I & $0.7-1.8$ & 0.493 \\
\hline \multicolumn{6}{|c|}{ Patients who were advised to reduce salt intake } \\
\hline & 122 [47.1\%] & $62[45.6 \%]$ & 0.7 & $0.6-1.4$ & 0.774 \\
\hline \multicolumn{6}{|c|}{ Patients who reported having their daily caloric needs calculated } \\
\hline & $12[4.6 \%]$ & $16[11.8 \%]$ & 2.7 & $1.2-5.9$ & 0.011 \\
\hline \multicolumn{6}{|c|}{ Patients who reported having their physical activity measured } \\
\hline & $6[2.3 \%]$ & $6[4.4 \%]$ & 1.9 & $0.6-6.1$ & 0.257 \\
\hline \multicolumn{6}{|c|}{ Patients who were advised to exercise } \\
\hline & 197 [76.1\%] & 113 [83.1\%] & 1.5 & $0.9-2.6$ & 0.108 \\
\hline \multicolumn{6}{|c|}{ Patients who reported being referred to smoking cessation clinic } \\
\hline & 8 [14.8\%] & $\mathrm{I}[3.4 \%]$ & 0.20 & $0.02-1.7$ & 0.145 \\
\hline
\end{tabular}

Notes: Bold ORs, $95 \% \mathrm{Cl}$ and $\mathrm{P}$ values indicate statistically significant associations.

oriented advice pertaining to the delivery of physical activity advice. Additionally, it can be noted that dietary assessment is reported more frequently among our sample of patients with diabetes in comparison to physical activity assessment. This suggests variability in provision of items pertaining to lifestyle counseling where more effort is devoted toward dietary counseling than physical activity counseling. Finally, among the 33 patients who reported being current smokers among the patients attending follow-up sessions at different healthcare facilities in the region, only 9 reported being referred to smoking cessation clinics.

The assessment of the statistical variation of the frequencies and proportions of the reported receipt of lifestyle counseling according to history of comorbidities, duration since diagnosis with diabetes and educational levels revealed no statistically significant difference except concerning the receipt of dietary advice pertaining to the reduction of salt intake and referral to smoking cessation clinics. Dietary advice delivery, especially concerning the reduction of salt intake, was higher among older illiterate patients with a history of comorbidities (p-values $<0.05$ ). Referral to a smoking cessation clinic was higher among younger patients with no comorbidities and less than ten years since their diagnosis with diabetes ( $\mathrm{p}$-values $<0.05$ ).

Table 4 illustrates the odds of receiving lifestyle counseling services among patients following up at PHCs to patients following up at other healthcare facilities. Only the ORs of receiving a lifestyle plan and having daily caloric needs calculated were higher among patients following up at other healthcare establishments in comparison to those following up at PHCs with statistically significant values ( $\mathrm{p}$-values $<0.05$ ). Additionally, a marginal statistical significance was observed concerning the higher ORs of receiving advice to lose weight among patients following up at other healthcare establishments ( $\mathrm{P}$ value of 0.06 ). 


\section{Discussion}

This cross-sectional investigation identified 461 patients diagnosed with diabetes in the Jazan region in southwest Saudi Arabia. Sixty-six patients reported not attending a follow-up at any healthcare facility. When the participants were asked how their disease was diagnosed, the majority indicated that the diagnosis was only made after seeking healthcare services due to the development of symptoms or complications related to diabetes, suggesting limited screening efforts for diabetes in the region.

In all the identified healthcare settings, the majority of the patients received health education, which was mostly provided by the treating physicians, indicating the limited role of nutritionists or health educationists. A higher proportion of patients following up at the diabetes center received lifestyle counseling services compared to those at other facilities. This suggests a variation in the engagement of healthcare professionals in providing lifestyle counseling for patients diagnosed with diabetes according to healthcare facility. In addition, the study identified the limited receipt of goal-oriented advice pertaining to the delivery of nutritional or physical advice, which is explained by the limited measurement of the patients' level of physical activity or dietary habits. Finally, receipt of dietary counseling services was more likely to be reported by the patients in comparison to physical activity counseling services.

The majority of the studies conducted in Saudi Arabia concerning diabetes lifestyle risk factors mainly assessed the distributions and patterns among populations with different characteristics. A consensus on the importance of modifying the lifestyle of Saudis to reduce their risk profile pertaining to diabetes and other metabolic illnesses was observed..$^{9,25,26}$ In a limited experimental study conducted among 59 women in the western region of Saudi Arabia where the intervention group received a designed program involving health education, exercise sessions, and diet counseling provided by health educationists, physiotherapists, and nutritionists, the findings indicated an improvement in reducing blood pressure and blood glucose levels. Furthermore, the importance of interdisciplinary programs to ensure appropriate lifestyle counseling was emphasized. ${ }^{27}$ Although the objective of our investigation does not involve an assessment of the adherence or impact of lifestyle counseling, it can be noted that according to our findings, most of the lifestyle counseling was provided by the treating physicians and an extremely limited role of health educationists and nutritionists was observed, especially among the attendees at the PHCs in the Jazan region. This indicates limited implementation of an interdisciplinary approach to enhance the provision of lifestyle counseling to patients with diabetes in Jazan.

Our study identified a limited receipt of lifestyle counseling services for patients diagnosed with diabetes. Although healthcare workers were not involved in the current investigation, it is possible to postulate that the adherence and attitude of these workers toward a healthy lifestyle may influence the involvement of those healthcare workers with the provision of lifestyle counseling to their patients. One factor that has been suggested to influence patients to adhere to a healthy lifestyle is having their treating physicians demonstrate the same. ${ }^{28}$ Additionally, an investigation conducted in the Jazan region involving 234 PHC physicians detected a high prevalence of overweight and obese physicians and limited engagement in a healthy lifestyle after measuring their physical activity and dietary habits. ${ }^{29}$ Similar observations were noted among healthcare workers in Riyadh in the eastern region of Saudi Arabia, indicating limited engagement in a healthy lifestyle among healthcare workers. $^{30,31}$ This highlights the importance of considering the influence of healthcare workers' attitudes toward and adherence to a healthy lifestyle and the possible consequences when providing lifestyle counseling to their patients with type 2 diabetes.

Our investigation identified 66 patients who were diagnosed with diabetes and who had stopped attending followups at any healthcare facility to monitor their clinical and laboratory parameters pertaining to diabetes. Though factors related to the lack of follow-up among this group of patients are not assessed in the current investigation, this observation implies a lack of utilization of the freely available healthcare services. This lack of utilization of healthcare services can be explained by several potential factors including, but not limited to, access to healthcare, and financial and psychological circumstances. Moreover, around one-third of the identified patients were illiterate or had lower levels of education, and it is expected that this group of patients will very likely require special considerations when providing lifestyle counseling and ensuring effective adherence.

Only a small number of the patients identified in our sample reported being diagnosed with diabetes as a result of a screening campaign. One explanation is the lack of a screening program for diabetes in Saudi Arabia. ${ }^{32}$ This indicates a need for large-scale programs that can ensure the early detection of cases and the provision of lifestyle 
counseling for individuals who already have diabetes or are at risk of developing it.

The limited receipt of lifestyle counseling identified in our study is consistent with the findings of similar international investigations. A large-scale retrospective database analysis of about 185 million healthcare visits of patients in the United States indicated that more than half of the visits of patients diagnosed with chronic diseases, including diabetes, did not include any type of diet or exercise counseling. Furthermore, among the patients who were identified as smokers, $78.6 \%$ did not receive any smoking cessation advice. ${ }^{15}$ This finding is similar to ours, in which about one-third of the patients who were smokers were referred to smoking cessation clinics.

A Canadian study by Baillot et al investigated physical activity assessment and counseling in primary care settings by recruiting physicians, nurses and a sample of 439 patients either diagnosed with chronic disease or attending regular follow up sessions. Baillot et al reported that nearly half of the identified patients had their physical activity level assessed but only $21.6 \%$ received physical activity counseling. ${ }^{19}$ It can be noted that their findings is different than the findings of our investigation where only 12 patients with diabetes $(2.6 \%)$ reported having their physical activity level measured indicating an extremely limited physical activity assessment among patients attending all healthcare settings in Jazan. It is possible to argue that the limited physical activity assessment identified in our investigation can be partially explained by the limited physical activity counseling training provided for healthcare workers in the region. This notion is similar to the conclusions of a systematic review by Wattanapisit et al suggesting a need for more physical activity counseling training in primary care residency programs. ${ }^{18}$

\section{Strengths and Limitations}

This investigation has multiple strengths and limitations. The main strength of this investigation is its reliance on interviews to ensure the recruitment of patients who are unable to participate in self-administered questionnaires and avoid possible selection bias. An additional strength is conducting the study in community settings, which enabled the identification of subjects who were not attending follow-ups at any healthcare facility. The main limitation of this study is the dependence on the reported practices of the patients rather than an objective measurement in clinical settings. Additionally, the non-random sampling utilized in this investigation is another limitation that might influence the ability to generalize the findings.

\section{Conclusion}

Among the identified cohort of patients with diabetes in the Jazan region, a small number benefited from screening services. Furthermore, a limited receipt of lifestyle counseling services at different healthcare facilities was observed. The majority of the counseling services were provided by physicians, which indicates the limited interdisciplinary involvement of other health professionals such as nutritionists and health educationists. There is scope for further investigations concerning the constraints on the utilization of lifestyle counseling services, either at administrative levels, such as targeting institutions and the relevant stakeholders in the Ministry of Health, or at the service providers' level, which includes physicians, nutritionists, and health educationists. Furthermore, identifying patients diagnosed with diabetes who do not follow up at any healthcare facilities suggests an area for investigation to understand the factors contributing to the lack of followup for disease management, such as psychological factors and issues related to healthcare access.

\section{Author Contributions}

The author contributed to data analysis, drafting or revising the article, gave final approval of the version to be published, agreed to the submitted journal, and agree to be accountable for all aspects of the work.

\section{Funding}

This research did not receive any specific grant from any funding agency.

\section{Disclosure}

The author reported no conflicts of interest for this work.

\section{References}

1. World Health Organization. Technical package for cardiovascular disease management in primary health care: healthy-lifestyle counselling; 2018. Available from: https://apps.who.int/iris/bitstream/handle/10665/ 260422/WHO-NMH-NVI-18.1-eng.pdf? sequence=1. Accessed July 9, 2021.

2. International Diabetes Federation. IDF diabetes atlas: ninth edition 2019; 2019. Available from: https://www.diabetesatlas.org/upload/ resources/material/20200302_133351_IDFATLAS9e-final-web.pdf.

Accessed January 16, 2021.

3. World Health Organization. The top 10 causes of death; 2018. Available from: https://www.who.int/news-room/fact-sheets/detail/thetop-10-causes-of-death. Accessed January 16, 2021.

4. Riddle MC, Herman WA. The cost of diabetes care-an elephant in the room. Diabetes Care. 2018;41(5):929-932. (1935-5548 (Electronic)). doi:10.2337/dci18-0012

5. Alhowaish AK. Economic costs of diabetes in Saudi Arabia. J Family Community Med. 2013;20(1):1-7. doi:10.4103/2230-8229.108174 
6. American Diabetes Association. 3. Prevention or delay of type 2 diabetes: standards of medical care in diabetes-2019. Diabetes Care. 2019;42(Suppl 1):S29-s33. doi:10.2337/dc19-S003

7. Saudi Ministry of Health. National diabetes prevention and control program: Saudi national reference for diabetes mellitus guidelines in primary health care; 2014. Available from: https://www.moh.gov.sa/Ministry/ About/Health\%20Policies/026.pdf. Accessed January 16, 2021.

8. International Diabetes Federation. IDF MENA members: Saudi Arabia; 2020. Available from: https://idf.org/our-network/regionsmembers/middle-east-and-north-africa/members/46-saudi-arabia. html. Accessed January 16, 2021.

9. Gosadi IM. Assessment of the environmental and genetic factors influencing prevalence of metabolic syndrome in Saudi Arabia. Saudi Med J. 2016;37(1):12-20. doi:10.15537/smj.2016.1.12675

10. Saudi Ministry of Health. Saudi Ministry of Health: health information survey; 2013. Available from: https://www.moh.gov.sa/Ministry/ Statistics/Documents/Final\%20book.pdf. Accessed January 16, 2021.

11. American Diabetes Association. 1. Improving care and promoting health in populations: standards of medical care in diabetes-2021. Diabetes Care. 2018;41(Supplement 1):S7-12. (1935-5548 (Electronic)). doi:10.2337/dc18-S001

12. LeRoith D, Biessels GJ, Braithwaite SS, et al. Treatment of diabetes in older adults: an endocrine society* clinical practice guideline. $J$ Clin Endocrinol Metab. 2019;104(5):1520-1574. (1945-7197 (Electronic)).

13. Zhang M, Zhou Y, Zhong J, Wang K, Ding Y, Li L. Current guidelines on the management of gestational diabetes mellitus: a content analysis and appraisal. BMC Pregnancy Childbirth. 2019;19(1):1-5. (1471-2393 (Electronic)).

14. Saudi Ministry of Health. Saudi national reference of clinical guidelines for care of diabetic patients; 2014. Available from: https://www. moh.gov.sa/en/Ministry/About/Health\%20Policies/009.pdf. Accessed January 16, 2021.

15. Heaton PC, Frede SM. Patients' need for more counseling on diet, exercise, and smoking cessation: results from the national ambulatory medical care survey. J Am Pharm Assoc (2003). 2006;46(3):364-369. doi:10.1331/154434506777069516

16. Jackowski RM, Pogge EK, Early NK, Fairman KA, Sclar DA. Provision of lifestyle counseling and the prescribing of pharmacotherapy for hyperlipidemia among us ambulatory patients: a national assessment of office-based physician visits. Am J Cardiovasc Drugs. 2018;18(1):65-71. doi:10.1007/s40256-017-0247-y

17. Noordman J, Koopmans B, Korevaar JC, van der Weijden T, van Dulmen S. Exploring lifestyle counselling in routine primary care consultations: the professionals' role. Fam Pract. 2013;30 (3):332-340. doi:10.1093/fampra/cms077

18. Wattanapisit A, Tuangratananon T, Thanamee S. Physical activity counseling in primary care and family medicine residency training: a systematic review. BMC Med Educ. 2018;18(1):159. doi:10.1186/ s12909-018-1268-1

19. Baillot A, Baillargeon JP, Paré A, Poder TG, Brown C, Langlois MF. Physical activity assessment and counseling in Quebec family medicine groups. Can Fam Physician. 2018;64(5):e234-e241.
20. Bartsch AL, Härter M, Niedrich J, Brütt AL, Buchholz A. A systematic literature review of self-reported smoking cessation counseling by primary care physicians. PLoS One. 2016;11(12): e0168482. doi:10.1371/journal.pone.0168482

21. Alhamdan AA, Alshammari SA, Al-Amoud MM, et al. Evaluation of health care services provided for older adults in primary health care centers and its internal environment. A step towards age-friendly health centers. Saudi Med J. 2015;36(9):1091-1096. doi:10.15537/ smj.2015.9.11789

22. Alahmed Z, Lobelo F. Physical activity promotion in Saudi Arabia: a critical role for clinicians and the health care system. J Epidemiol Glob Health. 2018;7(S1):S7-S15. doi:10.1016/j.jegh.2017.10.005

23. Jradi H. Awareness, practices, and barriers regarding smoking cessation treatment among physicians in Saudi Arabia. $J$ Addict Dis. 2017;36(1):53-59. doi:10.1080/10550887.2015.1116355

24. Karr S, Jackowski RM, Buckley KD, Fairman KA, Sclar DA. Cardiovascular risk factors and provision of lifestyle counseling for diabetes or prediabetes with comorbid obesity: analysis of office-based physician visits made by patients 20 years of age or older. Diabetes Spectr. 2019;32(1):53-59. doi:10.2337/ds18-0008

25. Alramadan MJ, Magliano DJ, Alhamrani HA, et al. Lifestyle factors and macro- and micro-vascular complications among people with type 2 diabetes in Saudi Arabia. Diabetes Metab Syndr. 2018;13 (1):484-91. (1878-0334 (Electronic)).

26. AlQuaiz AM, Siddiqui AR, Kazi AAO, Batais MA, Al-Hazmi AM. Sedentary lifestyle and Framingham risk scores: a Population-Based Study in Riyadh City, Saudi Arabia. BMC Cardiovasc Disord. 2019;19(1):1-11. (1471-2261 (Electronic)). doi:10.1186/s12872019-1048-9

27. Khouja JH, Al Jasir B, Bargawi AA, Kutbi M. Lifestyle intervention for cardiovascular disease risk factors in Jeddah, Saudi Arabia. Cureus. 2020;12(11):e11791.

28. Oberg EB, Frank E. Physicians' health practices strongly influence patient health practices. $J R$ Coll Physicians Edinb. 2009;39 (4):290-291. doi:10.4997/JRCPE.2009.422

29. Gosadi I, Daghriri K, Majrashi A, et al. Lifestyle choices and prevalence of chronic noncommunicable diseases among primary healthcare physicians in the Jazan Region, Saudi Arabia. J Fam Med Prim Care. 2020;9(11):5699-5704. doi:10.4103/jfmpc.jfmpc_1472_20

30. Al-Qahtani MF. Health-promoting lifestyle behaviors among nurses in private hospitals in Al-Khobar, Saudi Arabia. J Egypt Public Health Assoc. 2015;90(1):29-34. doi:10.1097/01. EPX.0000461325.97703.8a

31. Al Alwan I, Badri M, Al-Ghamdi M, Aljarbou A, Alotaibi H, Tamim H. Prevalence of self-reported cardiovascular risk factors among Saudi physicians: a Comparative Study. Int J Health Sci (Qassim). 2013;7(1):3-13. doi:10.12816/0006015

32. Gosadi IM. National screening programs in Saudi Arabia: overview, outcomes, and effectiveness. J Infect Public Health. 2019;12 (5):608-614. doi:10.1016/j.jiph.2019.06.001
Journal of Multidisciplinary Healthcare

\section{Publish your work in this journal}

The Journal of Multidisciplinary Healthcare is an international, peerreviewed open-access journal that aims to represent and publish research in healthcare areas delivered by practitioners of different disciplines. This includes studies and reviews conducted by multidisciplinary teams as well as research which evaluates the results or conduct of such teams or healthcare processes in general. The journal covers a very wide range of areas and welcomes submissions from practitioners at all levels, from all over the world. The manuscript management system is completely online and includes a very quick and fair peer-review system. Visit http://www.dovepress.com/testimonials. php to read real quotes from published authors. 